\title{
Protocol: systematic review of the association between socio-economic status and survival in adult head and neck cancer
}

\author{
Bilal G. Taib ${ }^{1 *} \mathbb{D}$, Joseph Rylands², Sue Povall ${ }^{3}$, Terry M. Jones ${ }^{4}$ and David Taylor-Robinson ${ }^{3}$
}

\begin{abstract}
Background: Head and neck cancer incidence is increasing worldwide. Despite overall improvements in survival, numerous studies suggest worse survival in more disadvantaged populations; however, this literature has not been systematically reviewed. The aim of this review is to investigate whether lower compared to higher socioeconomic status (SES) influences survival in head and neck squamous cell cancer (HNSCC) and explore possible explanations for any relationship found.
\end{abstract}

Method: A systematic strategy will be used to identify articles, appraise their quality and extract data. Online databases including MEDLINE, Web of Knowledge, ESBCO Host and Scopus will be used to locate observational studies of adults with a primary diagnosis of head and neck cancer in EU15+ countries (15 members of the EU, Australia, Canada, Norway, USA and New Zealand) where the outcomes report associations between SES and survival. This will be augmented by searching for grey literature and through reference lists. Data will be extracted using a standardised form. Study quality will be assessed using the Newcastle Ottawa scale and where possible meta-analysis of the pooled data will be conducted.

Discussion: This review will quantify the association between SES and survival outcomes for adult head and neck cancer patients in developed countries. The results will help identify gaps in the literature and therefore direct further novel research in the field. Ultimately, this will inform public policy and strategies to reduce the inequalities in HNSCC survival.

\section{Systematic review registration: PROSPERO CRD42016037019.}

Keywords: Head and neck cancer, Socio-economic status, Socio-economic inequalities, Survival, Systematic review, Meta-analysis

\section{Background}

A large body of literature on health inequalities shows that more disadvantaged populations are more likely to experience illnesses and premature death [1]. Socioeconomic inequalities have been demonstrated in head and neck cancer incidence (HANC) [2]. However, evidence on the relationship between social deprivation and HANC survival has not been comprehensively synthesised.

Collectively, head and neck cancer is the 6th most common cancer worldwide accounting for 550,000 new

\footnotetext{
* Correspondence: bgani56@gmail.com

'Postgraduate Centre, Royal Liverpool University Hospital, Prescot Street, Liverpool L7 8XP, UK

Full list of author information is available at the end of the article
}

cases per annum and this figure is rising [3, 4]. Overall and disease-specific survival for head and neck cancer patients has improved, particularly for human papillomavirus (HPV)-positive oropharyngeal cancer $[5,6]$. However, the full extent of this survival benefit has not been conferred to HPV-negative head and neck squamous cell carcinomas (HNSCC) which tend to affect a different cohort of patients $[7,8]$.

In 2013, over 8000 new cases of HNSCC were diagnosed in England and Wales with an overall mortality rate of 13.4\% [9]. The estimated cost of HANC hospitalisations in the NHS is around $£ 57.1$ million per annum [10]. This debilitating disease also impacts on daily life long after the initial diagnosis and treatment. In the 
USA, the loss of productivity, as measured by a loss of earnings, is estimated to be $\$ 3.4$ billion [11]. The economic burden and social burden of HANC falls hardest on those in lower socio-economic status (SES) groups.

There are several individual studies examining the link between SES and survival in head and neck cancer in general and or at specific subsites. Most suggest there is a strong association between low SES and worse survival outcomes [12-21]. Using Scottish registry data, Robertson et al. estimated that there was a $27 \%$ greater risk of death for those in the most deprived SES groups compared to the least [12]. Nutting et al. described that improvements in laryngeal cancer survival in England and Wales for men from 1985 to 2001 are limited to the most affluent groups [22]. Furthermore, both McDonald et al. and Rachet et al. have suggested that the absolute differences in survival between the most and least affluent groups appear to be increasing $[13,14]$.

Estimates of the impact of SES on health and neck cancer survival vary. This may be because studies have used different designs, measured SES in different ways and adjusted for different confounding variables (such as age, sex, race, stage, alcohol and tobacco consumption, region, income, education and occupation). For instance, a retrospective cohort study collating evidence from two oncology studies on non-metastatic head and neck cancer by Coyne et al. estimates that the lowest income groups have a $30 \%$ increased risk of HNSCC mortality whereas a similar study looking into neighbourhood deprivation in America and its effect on head and neck cancer outcomes published by Retizel et al. 2012 estimates this to be at $70 \%[20,23]$. In this review, we aim to synthesise the available evidence and to explore reasons for any differences uncovered across the included studies.

Our review is part of a larger project aimed at better understanding the relationship between SES and a range of HNSCC outcomes. Our analyses are informed by the Diderichsen model of pathways to inequalities in health (Fig. 1) [24]. In the model, an individual's social position leads to differential exposure and vulnerability to risk factors, for instance tobacco and alcohol in the case of HNSCC. These processes lead to differential health outcomes with varying levels of access to health care that may have differential social consequences on income and ultimately survival. Similar frameworks have been utilised to describe inequalities in cancer outcomes in breast, prostate, cervical and colorectal cancer [25, 26].

The first part of this pathway, the association between SES and the risk of developing HANC, has been explored in a systematic review by Conway et al. The review showed that people in the lowest educational and income groups had at least a twofold increased risk of developing HANC [2].

However, there has been no overarching systematic review of the evidence assessing the association between SES and survival outcomes in HNSCC, and only parts of the putative causal pathway (as per Fig. 1) have been explored. The aim of this systematic review is therefore 
to investigate whether lower compared to higher SES influences survival in HNSCC in developed countries and to explore the variables that may explain any relationship found. We will assess the magnitude, statistical significance and associations of the SES variables with survival. This will help identify any gaps in the current evidence base and allow us to focus our public health efforts to reduce the burden of HNSCC.

\section{Methods/design}

To improve the transparency and completeness of this systematic review protocol, a completed copy of the Preferred Reporting Items for Systematic Reviews and Meta-Analyses for Protocols 2015 (PRISMA-P 2015) checklist is available as an Additional file 1 [27].

\section{Research question}

For individuals from developed countries, does lower compared to higher socioeconomic status influence survival in head and neck cancer?

\section{Population}

Any individual including and over the age of 18, of any gender from a European Union 15+ (EU15+) country will be selected. A EU15+ country is any of the first 15 members of the EU and Australia, Canada, Norway, USA and New Zealand. A similar set of countries has been used in previous UK-based comparative health inequality studies [28].

\section{Outcome}

The primary outcome of interest is differences in HNSCC survival between SES groups. This aggregate level data is measured as univariate and multivariate odds ratios or hazard ratios with $95 \%$ confidence intervals.

\section{Exposure}

We will focus on SES measures at individual or aggregate level data by income, education, occupation and neighbourhood deprivation indices. SES levels will be measured as per Conway et al. using a five-level ordinal strata, if possible [29].

\section{Other data}

In addition, data on potentially confounding or mediating factors will be sought, such as alcohol and smoking, age, sex, marital status, co-morbidities, time to presentation, stage of presentation and types of surgical and or medical interventions.

\section{Inclusion/exclusion criteria}

The following studies will be included in the review:

- Observational studies (cross-sectional, ecological, case-control, cohort (prospective and retrospective) reporting quantitative results and analysis of empirical data on association between socioeconomic status and survival for patients with HNSCC (ICD C00-C14 and C30-C32).

- This can be measured at individual or aggregate level by occupation, income, education, employment or neighbourhood deprivation where the outcome is an odds ratio or hazards ratio with measures of variance.

o EU15+ countries.

- Publications written in or translated into English, reporting on human subjects and published from 1990, inclusive.

The following studies will be excluded from the review. Those focussing on:

○ Loco-regional recurrence of head and neck cancer

- Second primary HNSCC

- Outcomes for patients primarily presenting with cancers of the nasopharynx, thyroid or oesophagus

- Subjects $<18$ years of age

\section{Search strategy}

A systematic strategy will be used to identify relevant articles, assess their eligibility and appraise their quality. This will be achieved by searching databases, the reference lists for any studies accepted for inclusion and grey literature.

Electronic searching of four databases will be performed: MEDLINE, Web of Knowledge, ESBCO Host and Scopus. These databases were discussed within the research collaboration and were identified as the most likely to yield relevant papers. We will include data from the 20 economically developed EU 15+ nations outlined in Appendix 1.

The search terms outlined in Appendix 2 were piloted prior to selection. They comprise of specific head and neck cancers, inequality terms and developed countries of interest. Relevant terms were identified during an initial scoping literature review of the topic.

Where possible, terms will be exploded to widen the search. Terms will be added as keywords if they cannot be exploded or if the exploded terms are not relevant to the research question. Truncation and proximity operators will also be applied as necessary to broaden the search. For consistency, the same terms will be inputted for each database, e.g. Scopus and Web of Science; however, as the functionality of each database is different, the terms have been adapted for correct use in each.

The same filters will be applied to all included databases as per the inclusion criteria. This will ensure articles are in English, on human subjects and investigating the influence of SES on HNSCC from 1990 onwards. As 
social conditions change over time, limiting the literature to this time period will ensure that publications are as relevant as possible to the present day. The search will be broad as studies examining the incidence of HNSCC may also examine the impact of SES on survival as a secondary outcome. The searches will be re-run after the initial data extraction period so any further studies are included. The remaining publications will then be exported to a referencing software and combined so that any duplicates are removed. These will then be screened as per the eligibility criteria.

Titles, abstracts and full texts will be screened independently by one author (BGT). The second reviewer will screen a $10 \%$ sample of the extracted papers-at title, abstract and full text stages (SP) to ensure consistency in the application of the eligibility criteria. If it is not clear from the title or abstract whether analyses by SES have been performed, the full text of the article will be retrieved and examined. All full text studies will be reviewed independently by two reviewers (BGT and SP) to ensure that the studies meet the inclusion and exclusion criteria. Any discrepancies either at the title, abstract or full-text phase will be discussed (DTR and TMJ) until an agreement is reached between all reviewers.

Another strategy will consist of searching through the reference list of articles that may have been missed by electronic database searches. Studies of interest will have their titles and abstracts analysed and screened as per our inclusion and exclusion criteria. We will also use the forward citation map to locate newer articles which have cited the older study identified. The full text for those articles that meet the initial inclusion criteria will be screened (BGT and JR) and any discrepancies discussed between the reviewers.

Finally, we will further augment our search by searching for grey literature. We will do this by entering the terms "head and neck cancer", "socioeconomic", "social class" and "deprivation" into the Google internet search engine and Google Scholar search application and assessing the first 100 results including reports from cancer registries, HANC audit reports, published abstracts and theses. In a similar manner, we will also search OpenGrey a repository for grey literature in Europe. Again, this will be performed independently by two reviewers (BGT and JR), and any disagreements will be resolved through discussion with the wider group.

\section{Quality assessment}

Risk of bias and quality assessment will be conducted by two reviewers (BGT and JR). Methodological quality of the included studies will be assessed using the Newcastle-Ottawa Scale and/or Cochrane risk of bias framework [30, 31]. Any discrepancies identified will be discussed and reviewed by the review team.

\section{Data analysis and synthesis}

To organise this data and to aid comparison between studies, tables will be created from which extracted data will be placed in a standardised manner. A proforma has been developed and piloted on three studies. Information to be extracted includes: year of publication, country, hypothesis, study design, level of analysis, sample size, age, recruitment period, SES variable measured, health measure outcome(s), tool used to measure the outcome, covariates analyses, significant and non-significant results, adjusted and unadjusted odds ratio/hazard ratio (OR/HR) for all cause and HNSCC-specific mortality, confidence intervals, conclusions, comments on limitations and quality assessment. Extracted data will be checked by one other reviewer (JR). We are anticipating to be able to explore heterogeneity and undertake analyses to explore the impact of potentially moderating factors such as age, sex, performance status, site, alcohol consumption, smoking, grade, stage, treatment employed, SES measures and neighbourhood deprivation. Harvest plots maybe used where appropriate to display and summarise the results of the studies and to display the impact of moderating factors subgrouping graphically [32].

Where possible meta-analysis of extracted data will be undertaken via the Mantel-Haenszel method using a fixed-effects model as default, unless significant heterogeneity is present, in which case a random-effects model will be used. HRs will be calculated with $95 \%$ confidence intervals. A $p$ value of $<0.05$ will be regarded as significant. Heterogeneity will be assessed using the $I$-squared statistic. We will undertake sensitivity analysis on the basis of study quality.

\section{Dissemination}

The systematic review will be submitted for publication. The findings will be presented at national and international conferences. It will also be presented locally to help inform the council and public health initiatives.

\section{Discussion}

This protocol describes how this systematic review will determine the impact of SES on the survival outcomes of adult HNSCC patients. Using the Diderichsen model (Fig. 1), we will identify the key variables and pathways that generate inequalities in survival in HANC.

In the UK, the gender-specific inequality gaps in survival between certain HANCs are the widest of any cancer [14]. Furthermore, the incidence of HANC is increasing and stark geographical inequalities exist in the UK [29]. This, coupled with a recent survival benefit generally limited to the more affluent groups, highlights the necessity of better understanding of SES and HNSCC [22].

We hope that the results of this review will identify potential targets for intervention to reduce inequalities in HNSCC outcomes. 


\section{Appendix 1}

T1

Table 1 List of the EU $15+$ countries included in the study

\begin{tabular}{ll}
\hline European & Other countries \\
\hline Austria & Australia \\
Belgium & Canada \\
Denmark & New Zealand \\
Finland & Norway \\
France & USA \\
Germany & \\
Greece & \\
Ireland & \\
Italy & \\
Luxembourg & \\
Netherlands & \\
Portugal & \\
Spain & \\
Sweden & \\
UK
\end{tabular}

\section{Appendix 2}

Table 2 MEDLINE search strategy

\begin{tabular}{lll}
\hline Inequality & Head and neck cancers & Survival \\
\hline "Socioeconomic & "Head and Neck Neoplasms" [Mesh] & survival \\
Factors" [Mesh] & & \\
inequit* & "Carcinoma, squamous cell of head and & \\
& neck" [Supplementary Concept] & \\
inequalit* & $\begin{array}{l}\text { head AND neck AND (cancer OR } \\
\text { neoplasm OR carcinoma) }\end{array}$ & \\
&
\end{tabular}

("Socioeconomic Factors" [Mesh] OR inequit* OR inequalit* OR disparit*) AND ("Head and Neck Neoplasms"[Mesh] OR "Carcinoma, squamous cell of head and neck" [Supplementary Concept] OR (head AND neck AND (cancer OR neoplasm OR carcinoma)) AND survival); limited to $>1989$, human, abstract available, English language. Rows combined with logical "OR" and columns with logical "AND"

\section{Additional file}

Additional file 1: PRISMA-P checklist. (DOC $81 \mathrm{~kb}$ )

\section{Abbreviations}

HANC: Head and neck cancer; HNSCC: Head and neck squamous cell carcinomas; SES: Socio-economic status

\section{Acknowledgements}

We would like to thank the Liverpool Cancer Inequalities Research Network (LCIRN) team for sharing resources and knowledge in order to create this protocol.

\section{Funding}

Not applicable.

\section{Availability of data and materials}

Not applicable.

\section{Authors' contributions}

DTR, SP and TMJ conceived of the idea for this review. SP undertook a scoping review of inequalities in risk factors and outcomes for HNSCC. The database searches from the scoping review form the foundation of the searches for this review. BGT, JR and SP will be the primary reviewers of the articles and will conduct the data extraction for this systematic review. DTR and TMJ will contribute to data analysis and interpretation of the findings. BGT is the primary author of this manuscript; all authors have commented on drafts. All authors have read and approved the final manuscript.

Ethics approval and consent to participate

Not applicable.

\section{Consent for publication}

Not applicable.

\section{Competing interests}

The authors declare that they have no competing interests.

\section{Publisher's Note}

Springer Nature remains neutral with regard to jurisdictional claims in published maps and institutional affiliations.

\section{Author details}

${ }^{1}$ Postgraduate Centre, Royal Liverpool University Hospital, Prescot Street, Liverpool L7 8XP, UK. ${ }^{2}$ Aintree University Hospital, Longmoor Lane, Liverpool L9 7AL, UK. ${ }^{3}$ Institute of Psychology, Health and Society, University of Liverpool, Waterhouse building, Liverpool L69 3BX, UK. ${ }^{4}$ Department of Molecular and Clinical Cancer Medicine, University of Liverpool, 200 London Road, Liverpool L3 9GA, UK.

Received: 23 October 2016 Accepted: 18 July 2017

Published online: 02 August 2017

\section{References}

1. Wilkinson R, Marmot M. Social determinants of health: the solid facts. Geneva: World Health Organisation; 2003.

2. Conway DI, Brenner DR, McMahon AD, Macpherson LM, Agudo A, Ahrens W, et al. Estimating and explaining the effect of education and income on head and neck cancer risk: INHANCE consortium pooled analysis of 31 case-control studies from 27 countries. Int J Cancer. 2015;136(5):1125-39.

3. Jemal A, Bray F, Center MM, Ferlay J, Ward E, Forman D. Global cancer statistics. CA Cancer J Clin. 2011;61(2):69-90.

4. Parkin D, Whelan S, Ferlay J, Teppo L, Thomas D. Cancer incidence in five continents. Lyon: IARC Scientific Publications; 2003. VIII

5. Pulte $\mathrm{D}$, Brenner $H$. Changes in survival in head and neck cancers in the late 20th and early 21st century: a period analysis. Oncologist. 2010;15(9):994-1001.

6. Salazar CR, Anayannis N, Smith RV, Wang Y, Haigentz M Jr, Garg M, et al. Combined P16 and human papillomavirus testing predicts head and neck cancer survival. Int J Cancer. 2014;135(10):2404-12.

7. Pfister DG, Fury MG. New chapter in our understanding of human papillomavirus-related head and neck cancer. J Clin Oncol. 2014;32(30):3349-52.

8. Allison PJ. Factors associated with smoking and alcohol consumption following treatment for head and neck cancer. Oral Oncol. 2001;37(6):51320.

9. Healthcare Quality Improvement Partnership. National Head and Neck Cancer Audit 2013. 9th Edition. Leeds: Health and Social Care Information Centre.

10. Tempest MJ, Thurston S, Carroll SM, Keeping ST. PCN56 preliminary results from a study of hospital costs due to treatment of head and neck cancers in England. Value Health. 2012;15(7):A418-A9.

11. Bradley CJ, Yabroff KR, Dahman B, Feuer EJ, Mariotto A, Brown ML. Productivity costs of cancer mortality in the United States: 2000-2020. J Natl Cancer Inst. 2008;100(24):1763-70.

12. Robertson G, Greenlaw N, Bray CA, Morrison DS. Explaining the effects of socio-economic deprivation on survival in a national prospective cohort 
study of 1909 patients with head and neck cancers. Cancer Epidemiol. 2010;34(6):682-8.

13. McDonald JT, Johnson-Obaseki S, Hwang E, Connell C, Corsten M. The relationship between survival and socio-economic status for head and neck cancer in Canada. J Otolaryngol Head Neck Surg. 2014;43(1):2.

14. Rachet B, Quinn MJ, Cooper N, Coleman MP. Survival from cancer of the larynx in England and Wales up to 2001. Br J Cancer. 2008;99(S1):S35-S7.

15. Groome PA, Schulze KM, Keller S, Mackillop WJ, O'Sullivan B, Irish JC, et al. Explaining socioeconomic status effects in laryngeal cancer. Clin Oncol (R Coll Radiol). 2006;18(4):283-92.

16. Elwood JM, Pearson JC, Skippen DH, Jackson SM. Alcohol, smoking, social and occupational factors in the aetiology of cancer of the oral cavity, pharynx and larynx. Int J Cancer. 1984;34(5):603-12.

17. Ragin CC, Langevin SM, Marzouk M, Grandis J, Taioli E. Determinants of head and neck cancer survival by race. Head Neck. 2011;33(8):1092-8.

18. Miller CS, Henry RG, Rayens MK. Disparities in risk of and survival from oropharyngeal squamous cell carcinoma. Oral Surg Oral Med Oral Pathol Oral Radiol Endod. 2003;95(5):570-5.

19. Konski A, Berkey BA, Kian Ang K, Fu KK. Effect of education level on outcome of patients treated on radiation therapy oncology group protocol 90-03. Cancer. 2003;98(7):1497-503.

20. Reitzel LR, Nguyen N, Zafereo ME, Li G, Wei Q, Sturgis EM. Neighborhood deprivation and clinical outcomes among head and neck cancer patients. Health Place. 2012;18(4):861-8.

21. Mackillop WJ, Zhang-Salomons J, Groome PA, Paszat L, Holowaty E. Socioeconomic status and cancer survival in Ontario. J Clin Oncol. 1997;15(4):1680-9.

22. Nutting $\mathrm{CM}$, Robinson $\mathrm{M}$, Birchall $\mathrm{M}$. Survival from laryngeal cancer in England and Wales up to 2001. Br J Cancer. 2008;99(Suppl 1):S38-S9.

23. Coyne JC, Pajak TF, Harris J, Konski A, Movsas B, Ang K, et al. Emotional well-being does not predict survival in head and neck cancer patients: a Radiation Therapy Oncology Group study. Cancer. 2007;110(11):2568-75.

24. Diderichsen F, Evans $T$, Whitehead M. The social basis of disparities in health. In: Evans T, Whitehead M, Diderichsen F, Bhuiya A, Wirth M, editors. Challenging inequities in health: from ethics to action. New York: Oxford University Press; 2001.

25. Krieger N. Defining and investigating social disparities in cancer: critical issues. Cancer Causes Control. 2005;16(1):5-14.

26. Krieger N, Emmons KM, White KB. Cancer disparities: developing a multidisciplinary research agenda_-preface. Cancer Causes Control. 2005; 16(1):1-3.

27. Moher D, Shamseer L, Clarke M, Ghersi D, Liberati A, Petticrew M, et al. Preferred reporting items for systematic review and meta-analysis protocols (PRISMA-P) 2015 statement. Syst Rev. 2015:4(1):1-9. https:// systematicreviewsjournal.biomedcentral.com/articles/10.1186/2046-4053-4-1.

28. Newton JN, Briggs ADM, Murray CJL, Dicker D, Foreman KJ, Wang $\mathrm{H}$, et al. Changes in health in England, with analysis by English regions and areas of deprivation, 1990-2013: a systematic analysis for the Global Burden of Disease Study 2013. Lancet. 2015;386(10010):2257-74.

29. Conway DI, Brewster DH, McKinney PA, Stark J, McMahon AD, Macpherson LMD. Widening socio-economic inequalities in oral cancer incidence in Scotland, 1976-2002. Br J Cancer. 2007;96(5):818-20.

30. Wells G, Shea B, O'Connell D, Peterson J, Welch V, Losos M, et al. The Newcastle-Ottawa Scale (NOS) for assessing the quality of nonrandomised studies in meta-analyses. 2013. Available from: http://www.ohri.ca/ programs/dlinical_epidemiology/oxford.asp. Accessed 1 Aug 2017.

31. Higgins JPT, Altman DG, Gøtzsche PC, Jüni $P$, Moher D, Oxman AD, et al. The Cochrane Collaboration's tool for assessing risk of bias in randomised trials. BMJ. 2011:343:d5928.

32. Ogilvie D, Fayter D, Petticrew M, Sowden A, Thomas S, Whitehead M, et al. The harvest plot: a method for synthesising evidence about the differential effects of interventions. BMC Med Res Methodol. 2008;8(1):8.

\section{Submit your next manuscript to BioMed Central and we will help you at every step:}

- We accept pre-submission inquiries

- Our selector tool helps you to find the most relevant journal

- We provide round the clock customer support

- Convenient online submission

- Thorough peer review

- Inclusion in PubMed and all major indexing services

- Maximum visibility for your research

Submit your manuscript at www.biomedcentral.com/submit
Biomed Central 